\title{
Pauli's exclusion principle during gravitational collapse
}

\author{
Open Physics Collaboration*†
}

October 30, 2019

\begin{abstract}
We propose a discussion of Pauli's Principle of Exclusion during the gravitational collapse of a star in terms of its temperature.
\end{abstract}

keywords: Pauli's exclusion principle, gravitation collapse and energy condition

\section{Introduction}

1. What happens to the particles that obey Pauli's principle of exclusion during the gravitational collapse of a star from an energy standpoint?

\section{Pauli's exclusion principle during gravitational collapse}

2. Considering the gravitational collapse of a star, we conjecture a possible consequence of the increase in its volumetric density as the temperature decreases, overcoming the pressure of degeneration attributed to the fermionic particles described by Pauli's exclusion principle.

*All authors with their affiliations appear at the end of this paper.

†Corresponding author: thiagosousamoraes4@gmail.com | Join the Open Physics Collaboration 
3. Fermions (electrons, protons, and neutrons) are subject to the Pauli's exclusion principle (PEP).

4. The PEP controls the collapse of a star due to the pressure of degeneracy, where atoms are prevented from collapsing over each other [1].

5. As a result of PEP, two spin- $1 / 2$ fermions cannot simultaneously have the same quantum numbers [2,3].

6. The occupation number of fermions determined by PEP dictates that two identical fermions cannot simultaneously occupy the same quantum state, so if a fermionic particle has spin $m_{s}=+1 / 2$, the other will have $m_{s}=-1 / 2[2]$.

7. The equation describing the occupation number for the fermions depends on the exponential of the inverse of the temperature,

$$
\eta(E)=\frac{g(E)}{e^{\alpha+\frac{E}{k_{B} T}}+1},
$$

where $\eta(E)$ is the energy density of the particles, $g(E)$ is the number of configurations with energy $E$ by cubic centimeter, $\alpha$ is the factor of degeneration, which depends on the particles density, $E$ is the energy of the particle, $k_{B}$ is the Boltzmann constant, and $T$ is the absolute temperature $[2|4|$.

8. For low densities, $\alpha \gg 0$, and for very high densities, $\alpha \ll 0[4]$.

9. The volumetric density of a fluid $(\rho)$ increases as its temperature decreases.

10. Suppose that during the collapse of a star, the factor of degeneration $\alpha$ decreases with the increase of the density $\rho$ due to the decrease of the temperature $T$ [1, 2, 4,

11. In a star capable of generating a black hole, the gravitational force during the gravitational collapse makes the density of the star much 
higher than the pressure of degeneracy, generating a rupture in spacetime $|5-7|$.

12. As a consequence, fermions in the black hole are brought to a "new state."

\section{Final Remarks}

13. A "new state of matter" would probably mean a superposition of fermions and bosons.

14. More discussions about the surprising consequence of the so called hollow black hole can be found here $8-12$.

\section{Open Invitation}

Please review, add content, and be a co-author of this article. Join the Open Mathematics Collaboration. Send your contribution to thiagosousamoraes4@gmail.com.

\section{Ethical conduct of research}

This original work was pre-registered under the OSF Preprints [13], please cite it accordingly [14. This will ensure that researches are conducted with integrity and intellectual honesty at all times and by all means.

\section{References}

[1] TIMLIN, John. Neutron Degeneracy Pressure. Quantum Mechanics II, Spring 2013. 
[2] Ilya G. Kaplan. Pauli Exclusion Principle and its theoretical foundation. Institute, National Autonomous University of Mexico, Mexico-city. https://arxiv.org/ftp/arxiv/papers/1902/1902.00499.pdf

[3] MARCEL, Walter J. Introdução a estrutura e Evolução das estrelas. São Paulo: EdUSP, 2018.

[4] SAGET, Irina; HEMPEL, Matthias; GREINER, Carsten and Jürgen Schaffner-Bielich. Compact Stars for Undergraduates. Institut für Theoretische Physik, J. W. Goethe Universität. https://arxiv.org/abs/astro-ph/0506417v1

[5] Lobo, Matheus P. "A Hole in the Black Hole." OSF Preprints, 18 Apr. 2019. https://doi.org/10.31219/osf.io/js7rf

[6] Lobo, Matheus P. "The Tipping Point of Temperature During Black Hole Formation." OSF Preprints, 28 June 2019. https://doi.org/10.31219/osf.io/zrk7u

[7] Lobo, Matheus P. "The Interior of a Black Hole and the Void of Spacetime." OSF Preprints, 12 May 2019. https://doi.org/10.31219/osf.io/awfx8

[8] Lobo, Matheus P. "Hollow Black Holes." OSF Preprints, 12 Aug. 2019. https://doi.org/10.31219/osf.io/rewub

[9] Lobo, Matheus P. "Black Hole Universe: A Grand Cosmic Recycler and Big Bang Generator?." OSF Preprints, 12 Aug. 2019. https://doi.org/10.31219/osf.io/pbdn3

[10] Lobo, Matheus P. "Dark Matter and Bubbles of Void." OSF Preprints, 11 July 2019. https://doi.org/10.31219/osf.io/w7m3q

[11] Lobo, Matheus P. "The Metric Tensor Pullback." OSF Preprints, 14 May 2019. https://doi.org/10.31219/osf.io/puhzw 
[12] Lobo, Matheus P. "What Is the Meaning of the Pullback Schwarzschild Metric?." OSF Preprints, 22 May 2019. https://doi.org/10.31219/osf.io/xq2bw

[13] COS. Open Science Framework. https://osf.io

[14] Moraes, Tiago S. "Pauli's Exclusion Principle During Gravitational Collapse." OSF Preprints, 29 Aug. 2019. https://doi.org/10.31219/osf.io/m5wz7

\section{The Open physics Collaboration}

Tiago Sousa Moraes (lead author, thiagosousamoraes4@gmail.com), ${ }^{1}$ Matheus Pereira Lobo ${ }^{2}$

${ }^{1}$ Colégio Estadual Rui Barbosa (Tocantins, Brazil); ${ }^{2}$ Federal University of Tocantins (Brazil) 